\title{
Gadolinium accumulation after contrast-enhanced magnetic resonance imaging: what rheumatologists should know
}

\author{
Fabio Martino Doniselli ${ }^{1}$ - Domenico Albano ${ }^{2} \cdot$ Vito Chianca $^{3}$. \\ Marco Amedeo Cimmino ${ }^{4}$. Luca Maria Sconfienza ${ }^{5,6}$ (D)
}

Received: 27 February 2017 / Accepted: 13 March 2017 / Published online: 20 March 2017

(C) International League of Associations for Rheumatology (ILAR) 2017

Keywords Gadolinium · Gadolinium storage condition · Magnetic resonance imaging $\cdot$ Rheumatoid arthritis .

Seronegative spondyloarthropathy

Magnetic resonance imaging (MRI) is an imaging modality capable to explore all anatomic areas of the human body, with the great advantage of avoiding ionizing radiation exposure. In the joints, MRI is the modality of choice in the evaluation of cartilage, joint space, and synovium and is excellent in evaluating all periarticular structures. MRI has gained an increased importance in rheumatology, mainly for the possibility of early diagnosis and evaluation of disease activity in patients with seronegative spondyloarthropaties involving the spine [1]. In addition, a baseline evaluation of the hand and wrist in patients with rheumatoid arthritis (RA) is considered useful by many rheumatologists because it can evaluate synovitis, ero-

Luca Maria Sconfienza

io@lucasconfienza.it

1 Postgraduate School in Radiodiagnostics, University of Milano, Via Festa del Perdono 7, 20122 Milan, Italy

2 Department of Radiology, DIBIMED, University of Palermo, Via del Vespro 129, 90127 Palermo, Italy

3 Department of Advanced Biomedical Sciences, Federico II University, Via Pansini 5, 80131 Naples, Italy

4 Research Laboratory and Academic Division of Clinical Rheumatology, Department of Internal Medicine, University of Genoa, Viale Benedetto XV 6, 16132 Genoa, Italy

5 Department of Biomedical Sciences for Health, University of Milano, Via Festa del Perdono 7, 20122 Milan, Italy

6 Unit of Diagnostic and Interventional Radiology, IRCCS Istituto Ortopedico Galeazzi, Via Riccardo Galeazzi 4, 20161 Milan, Italy sions, and bone marrow edema (BME) with a single examination [2]. The European League against Rheumatism (EULAR) recommendations on imaging in RA suggest that MRI can be useful to: confirm the diagnosis of RA by showing synovitis in clinically unaffected joints; predict the severity and progression of RA; and assess residual synovitis in RA patients in clinical remission [3]. The sensitivity in detecting erosions of the RA wrist is higher for MRI than for radiography. As a result, MRI has been accepted by both the Outcome Measures in Rheumatology (OMERACT) [4] and the American College of Rheumatology [5] for the evaluation of structural damage in clinical trials studying novel therapeutic agents in RA and in psoriatic arthritis. BME is a significant predictor of negative prognosis in patients with early, undifferentiated arthritis and in those with established RA [6]. Finally, synovitis can be visualized comparing unenhanced and contrast-enhanced static images but also by dynamic MRI, i.e., following the course of contrast enhancement during the time after injection [7]. All the previous aspects are summarized in a composite score, the rheumatoid arthritis MRI scoring system (RAMRIS), which is used during MRI follow-up of RA patients [8]. In the real world, few patients outside industry-sponsored clinical trials to test the efficacy of new drugs perform a regular follow-up by contrast-enhanced MRI. However, since very often the standards employed in controlled clinical trials are subsequently transferred to everyday clinical practice [9], it is possible that MRI will be more frequently used in RA in the next future.

Using static and variable magnetic fields and non-ionizing radiofrequency radiations, MRI has been traditionally regarded as a safe imaging modality in rheumatology, only limited by the presence of absolute contraindications such as the presence of MR-unsafe devices in patient's body. Contrast agents used in MRI are mainly based on paramagnetic substances, capable to locally change the relaxation times of 
tissues where they are concentrated after intravenous injection. The most used contrast agents are metal ion-ligand complexes, composed of a gadolinium ion $\left(\mathrm{Gd}^{3+}\right)$ bond with a chelating agent; $\mathrm{Gd}^{3+}$ shortens the longitudinal relaxation time of water protons [10]. The chelating ligand overcomes the direct toxicity by free $\mathrm{Gd}^{3+}$ maintaining its contrast properties and influences the biodistribution of the drug. Gadoliniumbased contrast agents (GBCAs) may have different chemical structures with different degrees of stability of the Gdchelating agent complex [11]. Initially, GBCAs were introduced in clinical practice to improve the detection and diagnosis of central nervous system diseases; only later, their utility has been recognized and approved in other fields, including not only oncologic imaging all over the body but also the evaluation of chronic and acute inflammatory conditions [12].

Since their introduction in the 1980s, GBCAs were administered with few restrictions and with the awareness of minimal patients' risk [13]. Nevertheless, 10 years ago, the identification of nephrogenic systemic fibrosis (NSF) as a possible non-allergic adverse effect of GBCAs administration to patients with renal impairment changed the clinical practice [14]. NSF is a rare disease characterized by skin thickening and fibrotic changes in various organs, ranging from mild clinical features to fatal conditions [14, 15]. The GBCAs with greatest risk of NSF have been reported to be gadopentetate, gadodiamide, and gadoversetamide. To date, all these GBCAs are contraindicated in patients with estimated glomerular filtration rate (eGFR) $<30 \mathrm{~mL} / \mathrm{min} / 1.73^{-\mathrm{m} 2}$, while the other GBCAs should be administered with caution [16]. These recommendations have substantially reduced to zero the new cases of NSF over the past few years.

Although the presence of renal impairment is a necessary requirement for the occurrence of NSF, $\mathrm{Gd}^{3+}$ accumulation in human tissues has been well demonstrated in patients with normal renal function. Evidences about $\mathrm{Gd}^{3+}$ accumulation after multiple injections of GBCAs has been known for 10 years, being described first in the bone using mass spectroscopy on hip specimens taken during hip replacement surgery [17]. However, $\mathrm{Gd}^{3+}$ accumulation in the bone has not been reported in vivo so far. Only in the last 3 years, $\mathrm{Gd}^{3+}$ deposition became a hot topic, when $\mathrm{Gd}^{3+}$ accumulation has been described in intracranial structures. First evidences of $\mathrm{Gd}^{3+}$ deposition in brain were reported in studies on MRI by Kanda et al. [18] and Errante et al. [19], and later confirmed by different groups [20]. Gadopentetate dimeglumine and gadodiamide were the molecules most frequently associated with signal increase in brain nuclei. Studies reported that this effect can be appreciated after about five injections of the recommended dose of $0.1 \mathrm{mmol} / \mathrm{kg}$.

Beginning from the qualitative clue of a signal intensity increase in cerebellar dentate nucleus, these authors discovered a statistically significant quantitative change on T1weighted images after multiple GBCAs administrations, not only in dentate nucleus but also in globus pallidus and other brain structures. So far, it is not clear in which chemical form $\mathrm{Gd}$ is accumulated.

Several factors may influence $\mathrm{Gd}^{3+}$ release, occurring most likely through a process of "transmetallation" induced by the presence of metals with high affinity for $\mathrm{Gd}^{3+}$ such as $\mathrm{Fe}^{3+}$, $\mathrm{Ca}^{2+}, \mathrm{Zn}^{2+}$, or ligands such as phosphate [21]. Despite attempts to find a common mechanism of deposition have failed, massspectroscopy analysis on autoptic specimens of patients who underwent multiple GBCA injection demonstrated the presence of variable $\mathrm{Gd}^{3+}$ levels in various human tissues, especially the bones, kidneys, skin, and brain [20, 22]. So far, $\mathrm{Gd}^{3+}$ accumulation in the brain has been mainly demonstrated only in the brain of patients undergoing MRI scans for the diagnosis of neurological diseases. Research is needed for evaluating $\mathrm{Gd}^{3+}$ accumulation in the brain of patients undergoing multiple contrast-enhanced MRIs for non-neurological purposes, such as rheumatologic patients. In fact, while $\mathrm{Gd}^{3+}$ brain accumulation in patients with neurological diseases may be related to a blood-brain barrier damage (frequent in diseases such as multiple sclerosis), studies showed GBCA effect related to $\mathrm{Gd}^{3+}$ accumulation also after excluding patients with potential bloodbrain barrier damage [23]. Thus, it can be argued that all patients undergoing multiple administrations of GBCAs are at risk of $\mathrm{Gd}^{3+}$ accumulation in the brain, regardless of the underlying pathology or anatomical district being investigated using MRI.

Studies in the last year tried to differentiate gadolinium deposition on the basis of the grade of dissociation from the ligand. Macrocyclic GBCAs have been shown both in vitro [24] and in vivo [25] to have lower releasing rate compared with linear ones, and could produce less deposition in human tissues.

On the other hand, the clinical significance of $\mathrm{Gd}^{3+}$ accumulation is still unclear. Various potential mechanisms of $\mathrm{Gd}^{3+}$ accumulation toxicity have been postulated, including the induction of release of profibrotic and proinflammatory chemokines and cytokines, oxidative stress, transmetallation, and competition of $\mathrm{Gd}^{3+}$ with $\mathrm{Ca}^{2+}$ [26]. To date, few clinical effects have been demonstrated, especially nephro-, hematoand neuro-toxicity on animal studies, frequently using very high doses, but only few case reports of neuro- and nephrotoxicity on humans have been published [26]. Recently, Semelka et al. evaluated the clinical aspects of $\mathrm{Gd}^{3+}$ accumulation in patients with normal renal function and termed this occurrence as "gadolinium storage condition". They reported that patients might experience skin thickening and bone/chest pain after administration of GBCAs [27]. They thus identify a new pathologic entity defined as "gadolinium deposition disease", which may share clinical features with NSF but also present with other, non-typical symptoms [28]. It is interesting to note that awareness of patients on the topic is increasing over time, as several patient advocacy groups on "gadolinium toxicity" have been recently established in the USA [29]. 
Future studies should be focused on possible clinical consequences of multiple GBCAs administrations and options to reduce $\mathrm{Gd}^{3+}$ accumulation. N-acetyl-cysteine or iron chelation pre-treatments have been proposed for reducing $\mathrm{Gd}^{3+}$ toxicity [26]. Furthermore, alternative options for the quantitative and objective assessment of joint inflammation with MRI avoiding GBCA injection such as diffusion-weighted imaging, T1 and T2 mapping, and MRI-ultrasound-Doppler fusion images may be considered [30].

In conclusion, MRI with the intravenous administration of GBCAs is useful in the assessment of rheumatologic conditions. So far, $\mathrm{Gd}^{3+}$ accumulation in the brain has not been associated with any clinical consequences. However, the rheumatologic community should be aware of the potential risks of repeated administrations of GBCAs.

Compliance with ethical standards No funding was received for the present paper. Ethical approval was not required for the present paper, as it does not involve any patients.

Disclosure None.

\section{References}

1. Sieper J, Poddubnyy D (2017) Axial spondyloarthritis. Lancet doi: 10.1016/S0140-6736(16)31591-4.

2. D'Agostino MA, Haavardsholm EA, van der Laken CJ (2016) Diagnosis and management of rheumatoid arthritis; What is the current role of established and new imaging techniques in clinical practice? Best Pract Res Clin Rheumatol 30(4):586-607. doi:10. 1016/j.berh.2016.10.011

3. Colebatch AN, Edwards CJ, Østergaard M et al (2013) EULAR recommendations for the use of imaging of the joints in the clinical management of rheumatoid arthritis. Ann Rheum Dis 72(6):804814. doi:10.1136/annrheumdis-2012-203158

4. Peterfy C, Strand V, Tian L, et al (2017) Short-term changes on MRI predict long-term changes on radiography in rheumatoid arthritis: an analysis by an OMERACT Task Force of pooled data from four randomised controlled trials. Ann Rheum Dis. doi:10. 1136/annrheumdis-2016-210311. [Published online first]

5. (2013) American College of Rheumatology Clinical Trials task Force Imaging Group and Outcome Measures in Rheumatology Magnetic Resonance Imaging Inflammatory Arthritis Working Group. The utility of magnetic imaging for assessing structural damage in randomized controlled trials in rheumatoid arthritis. Arthritis Rheum. 65(10):2513-23. doi:10.1002/art.38083

6. Nakashima Y, Tamai M, Kita J et al (2016) Magnetic resonance imaging bone edema at enrolment predicts rapid radiographic progression in patients with early rheumatoid arthritis: results from the Nagasaki University early arthritis cohort. J Rheumatol 43(7): 1278-1284. doi:10.3899/jrheum.150988

7. Boesen M, Kubassova O, Bouert R et al (2012) Correlation between computer-aided dynamic gadolinium-enhanced MRI assessment of inflammation and semi-quantitative synovitis and bone marrow oedema scores of the wrist in patients with rheumatoid arthritis - a cohort study. Rheumatology (Oxford) 51:134-143. doi:10.1093/rheumatology/ker220

8. Østergaard M, Peterfy C, Conaghan P et al (2003) OMERACT rheumatoid arthritis magnetic resonance imaging studies. Core set of MRI acquisitions, joint pathology definitions, and the OMERACT RA-MRI scoring system. J Rheumatol 30(6):13851386

9. Peterfy CG (2001) Magnetic resonance imaging of rheumatoid arthritis: the evolution of clinical applications through clinical trials. Semin Arthritis Rheum 30(6):375-396. doi:10.1053/sarh.2001. 22497

10. De León-Rodríguez LM, Martins AF, Pinho MC et al (2015) Basic MR relaxation mechanisms and contrast agent design. J Magn Reson Imaging 42(3):545-565. doi:10.1002/jmri.24787

11. Fraum TJ, Ludwig DR, Bashir MR, et al (2017) Gadolinium-based contrast agents: A comprehensive risk assessment. J Magn Reson Imaging. doi:10.1002/jmri.25625

12. Geraldes CFGC, Laurent S (2010) Classification and basic properties of contrast agents for magnetic resonance imaging. Contrast Media Mol Imaging 4(1):1-23. doi:10.1002/jmri.25625

13. Lautin EM, Freeman NJ, Schoenfeld AH et al (1991) Radiocontrast-associated renal dysfunction: incidence and risk factors. AJR Am J Roentgenol 157(1):49-58

14. Daram SR, Cortese CM, Bastani B (2005) Nephrogenic fibrosing dermopathy/nephrogenic systemic fibrosis: report of a new case with literature review. Am J Kidney Dis 46(4):754-759. doi:10. 1053/j.ajkd.2005.06.024

15. Todd DJ, Kagan A, Chibnik LB et al (2007) Cutaneous changes of nephrogenic systemic fibrosis: predictor of early mortality and association with gadolinium exposure. Arthritis Rheum 56(10):34333441. doi:10.1002/art.22925

16. Thomsen HS, Morcos SK, Almen T et al (2013) Nephrogenic systemic fibrosis and gadolinium-based contrast media: updated ESUR contrast medium safety committee guidelines. Eur Radiol 23(2): 307-318. doi:10.1007/s00330-012-2597-9

17. White GW, Gibby WA, Tweedle MF (2006) Comparison of Gd (DTPA-BMA) (Omniscan) versus Gd (HP-DO3A) (ProHance) relative to gadolinium retention in human bone tissue by inductively coupled plasma mass spectroscopy. Investig Radiol 41(3):272-278. doi:10.1097/01.rli.0000186569.32408.95

18. Kanda T, Ishii $\mathrm{K}$, Kawaguchi $\mathrm{H}$ et al (2014) High signal intensity in the dentate nucleus and globus pallidus on unenhanced T1weighted MR images: relationship with increasing cumulative dose of a gadolinium-based contrast material. Radiology 270(3):834841. doi:10.1148/radiol.13131669

19. Errante Y, Cirimele V, Mallio CA et al (2014) Progressive increase of $\mathrm{T} 1$ signal intensity of the dentate nucleus on unenhanced magnetic resonance images is associated with cumulative doses of intravenously administered gadodiamide in patients with normal renal function, suggesting dechelation. Investig Radiol 49(10):685690. doi:10.1097/RLI.0000000000000072

20. Kanda T, Fukusato T, Matsuda M et al (2015) Gadolinium-based contrast agent accumulates in the brain even in subjects without severe renal dysfunction: evaluation of autopsy brain specimens with inductively coupled plasma mass spectroscopy. Radiology 276(1):228-232. doi:10.1148/radiol.2015142690

21. Aime S, Caravan P (2009) Biodistribution of gadolinium-based contrast agents, including gadolinium deposition. J Magn Reson Imaging 30(6):1259-1267. doi:10.1002/jmri.21969

22. Murata N, Gonzalez-Cuyar LF, Murata K et al (2016) Macrocyclic and other non-group 1 gadolinium contrast agents deposit low levels of gadolinium in brain and bone tissue. Investig Radiol 51(7):447-453. doi:10.1097/RLI.0000000000000252

23. Roccatagliata L, Vuolo L, Bonzano L et al (2009) Multiple sclerosis: hyperintense dentate nucleus on unenhanced T1-weighted MR images is associated with the secondary progressive subtype. Radiology 251(2):503-510. doi:10.1148/radiol.2511081269

24. Frenzel T, Lengsfeld P, Schirmer H et al (2016) Stability of gadolinium-based magnetic resonance imaging contrast agents in 
human serum at 37 degrees C. Investig Radiol 43(12):817-828. doi:10.1097/RLI.0b013e3181852171

25. Radbruch A (2016) Are some agents less likely to deposit gadolinium in the brain? Magn Reson Imaging 34(10):1351-1354. doi:10. 1016/j.mri.2016.09.001

26. Rogosnitzky M, Branch S (2016) Gadolinium-based contrast agent toxicity: a review of known and proposed mechanisms. Biometals 29(3):365-376. doi:10.1007/s10534-016-9931-7

27. Semelka RC, Commander CW, Jay M, Burke LM, Ramalho M (2016) Presumed gadolinium toxicity in subjects with normal renal function: a report of 4 cases. Investig Radiol 51(10):661-665. doi: 10.1097/RLI.0000000000000318
28. Semelka RC, Ramalho J, Vakharia A et al (2016) Gadolinium deposition disease: initial description of a disease that has been around for a while. Magn Reson Imaging 34(10):1383-1390. doi:10.1016/ j.mri.2016.07.016

29. Burke LM, Ramalho M, AlObaidy M et al (2016) Self-reported gadolinium toxicity: a survey of patients with chronic symptoms. Magn Reson Imaging 34(8):1078-1080. doi:10.1016/j.mri.2016. 05.005

30. Potter HG, Black BR, Chong LR (2009) New techniques in articular cartilage imaging. Clin Sports Med 28(1):77-94. doi:10.1016/ j.csm.2008.08.004 